This item was submitted to Loughborough's Research Repository by the author.

Items in Figshare are protected by copyright, with all rights reserved, unless otherwise indicated.

\title{
The effects of licence disqualification on drink-drivers: Is it the same for everyone?
}

\section{PLEASE CITE THE PUBLISHED VERSION}

https://doi.org/10.1016/j.aap.2017.07.028

\section{PUBLISHER}

(C) Elsevier

VERSION

AM (Accepted Manuscript)

\section{PUBLISHER STATEMENT}

This work is made available according to the conditions of the Creative Commons Attribution-NonCommercialNoDerivatives 4.0 International (CC BY-NC-ND 4.0) licence. Full details of this licence are available at: https://creativecommons.org/licenses/by-nc-nd/4.0/

\section{LICENCE}

CC BY-NC-ND 4.0

\section{REPOSITORY RECORD}

Watson, Angela, James Freeman, K. Imberger, Ashleigh J. Filtness, Hollie Wilson, David Healy, and A. Cavallo. 2019. "The Effects of Licence Disqualification on Drink-drivers: Is It the Same for Everyone?". figshare. https://hdl.handle.net/2134/28452. 


\section{The effects of licence disqualification on drink-drivers: Is it the same for everyone?}

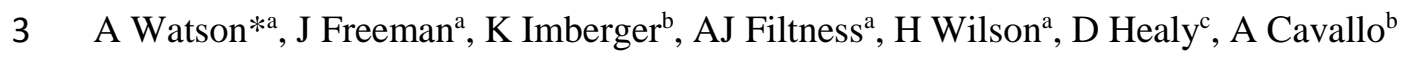

$4 \quad{ }^{a}$ Queensland University of Technology (QUT), Centre for Accident Research and Road Safety

5 - Queensland (CARRS-Q)

$6 \quad$ b VicRoads

$7 \quad$ c David Healy Road Safety Consulting

8 *Corresponding author:

9 Angela Watson, Ph.D.

10 CARRS-Q, Queensland University of Technology, K block, 130 Victoria Park Road, Kelvin

11 Grove, Queensland, 4059, Australia

12 Tel: +61731384565

$13 \quad$ Fax: +61 731380111

14 Email: angela.watson@qut.edu.au

15 


\section{Abstract}

Drink-driving remains a major road safety concern that creates a significant social burden. Licence disqualification continues to play a key role in drink driving deterrence and sanctions together with police enforcement to address the problem in most motorised countries. However, on-going questions remain regarding the differing effect of licence disqualification periods between first time and repeat offenders, and between other sub-groups of offenders. As a result, this study aimed to determine whether: (a) differences exist in re-offence rates of convicted drink-drivers between: the period between committing the drink-driving offence and licence disqualification (pre-licence disqualification), during the period of licence disqualification, and after being relicensed (post-licence restoration); and (b) differential effects of offence rates are evident based on Blood Alcohol Content (BAC), gender, age, repeat offender status and crash involvement at the time of offence. The sample consisted of 29,204 drink-driving offenders detected in Victoria, Australia between 1 January 1996 and 30 September 2002. The analysis indicated that licence disqualifications were effective as drinkdriving offenders had a significantly lower rate of offending (both drink-driving and other traffic offences) during licence disqualifications compared to pre-licence disqualification and post-licence restoration periods. The influence of licence disqualification appeared to extend beyond the disqualification period, as offence rates were lower during post-licence restoration than during pre-licence disqualification. Interestingly, the highest rate of offending (both for drink-driving and other traffic offences) was during the pre-licence disqualification period, which suggests offenders are particularly vulnerable to drink and drive while waiting to be sanctioned. A consistent pattern of results was evident across genders and age groups. Additionally, 
those who were involved in a crash at the same time as their index offence had lower

41 offence rates (compared to those who were not involved in a crash) for all periods,

42 although for general traffic offences, the offence rate was highest in the post-licence

43 restoration period for those who had a crash at index offence. This indicates that being

44 involved in a crash may deter these offenders, at least in the short-term. The

45 implications of the results for managing both first time and repeat offenders are

46 discussed.

47 Keywords: drink-driving, drunk-driving, licence disqualification, sanctions, offences

$48 \quad$ Highlights

- 6.5 years of offence history data for 29,204 drink-driving offenders was considered.

- Licence disqualification was effective at reducing drink-driving offence rates, as well as reducing general traffic offences.

- Licence disqualification had residual benefits as offence rates were lower postthan pre-disqualification.

- Offences were most prevalent in the lag time between offence and application of sanction.

\section{Introduction}

Drink-driving continues to be a serious and persistent problem in all motorised jurisdictions, as alcohol-related crashes result in substantial fatalities, injuries and property damage. Alcohol-related crashes are one of the leading causes of death on the roads, for example in Victoria, Australia 32\% of driver fatalities between 2008 and 2011 had a Blood Alcohol Concentration (BAC) over zero. In fact, 28\% of driver 
fatalities had an illegal BAC $(\geq .05)$ and $10 \%$ had a BAC over .2. Nearly $23 \%$ of motorcyclist fatalities had a BAC over zero (18\% of motorcyclist fatalities had an illegal BAC ( $\geq .05)$ and 4\% had a BAC over .2) (Coroners Prevention Unit, 2013). The legal BAC limit in Victoria is less than .05. Of particular concern is the proportion of repeat drink-driving offenders, for example within Victoria 30\% of detected drinkdrivers had a previous drink-drive conviction (Boorman, 2012). In regards to crashes, research has also demonstrated that repeat offenders are disproportionately represented in crash statistics (Beirness, Mayhew, \& Simpson, 1997; Brewer et al., 1994).

The gravity of the problem is reflected in the enormous amount of literature that has focused on the personal and economic cost of drink-driving, as well as the development and implementation of various countermeasures to reduce the prevalence of the offending behaviour (Beirness et al., 1997). Countermeasures to address drink-driving vary across different jurisdictions, although licence disqualification has historically formed the foundation of many legislative responses to such offending behaviours. The application of licensing sanctions has consistently proven an effective general and specific deterrent (Peck, 1991; Ross, 1991), although questions remain as to whether the sanction improves general driving behaviour for offenders post relicensing. General and specific deterrence stem from the Classical Deterrence Doctrine, which remains the mostly widely cited model for the study of sanctions effect(s) within road safety (Freeman et al., 2015). Specific deterrence is the process whereby an individual who has been apprehended and punished for a criminal act refrains from further offending behaviour for fear of incurring additional punishment (Homel, 1988). This phenomenon will remain the primary focus of the current study, in particular, the effect of licence disqualification. 
87 While there has been considerable focus on the impact of sanctions (Wagenaar, \& Maldonado-Molina, 2007), there has been limited consideration as to whether apprehended drink-drivers re-offend during the period of time between apprehension and application of sanction, despite waitlisting times to appear in court often being long (e.g. six to twelve months on average). However, it is noted that some preliminary research has focused on the positive impact of changes to administrative suspension laws that has resulted in a reduction in the penalty application timeframe (McArthur, \& Kraus, 1999; Voas, Tippets \& Fell, 2000). What is known is that drink-drivers are not a homogenous group (Nochajski \& Wieczorek, 2000), as research has demonstrated that first time and repeat offenders often differ in both characteristics and treatment needs (Stewart, Boase, \& Lamble, 2004). These two groups display a tendency to respond differently to the application of sanctions (Ferguson, Sheehan, Davey, \& Watson, 1999; Freeman, 2004), in particular, Norther American research has demonstrated that the application of licence sanctions on repeat offenders (in isolation) is relatively ineffective (Beirness et al., 1997; Coben \& Larkin, 1999).

An important consideration for the current study was to not only identify the effectiveness of licence disqualification, but also to assess the impact of this approach on different groups of offenders. Currently, questions also remain regarding the impact of licence disqualification periods on gender, age and BAC level at time of apprehension. That is, whether motorists respond differently to the sanction depending on their gender, age and level of alcohol consumption. Therefore, the project focuses on drink-driving outcome data and also considers the general demographics of the population (e.g., age, sex, drink-driving history). Without such a comprehensive 
111 investigation, a deeper understanding into the specific impact of licence sanction on re-

112 offence rates cannot be achieved. This project considers all facets in order to maximise

113 the potential to obtain large safety gains through the on-going sanctioning of drink-

114 drivers.

115 The aims of this study were to determine whether:

For each offender, the index drink-driving offence between 1 January 1996 and 30

\section{Method}

Drivers and riders convicted of a drink-driving offences committed between

1 January 1996 and 30 September 2002 (inclusive) were considered eligible persons for analysis $(\mathrm{N}=29,204)$. The time period was determined as part of a larger project to coincide with a period prior to alcohol ignition interlocks coming into effect. This was so that the unique effect of licence disqualification (without the influence of interlocks) could be assessed. Data files relating to all offences, licence status changes, disqualifications from driving, licence conditions, and driver and rider demographics were provided from the VicRoads Driver Licensing System (DLS).

September 2002 (the first drink-driving offence recorded) was identified. Offence rates were calculated for the period between the index offence and the licence disqualification 
134 (pre-licence disqualification period), the licence disqualification period, and the post-

135 licence restoration period. The rates of offences (drink-driving and other traffic

136 offences) were calculated per thousand person-years for all the licence/sanction periods.

137 This approach was based on previous research by Siskind (1996) to account for the

138 different length of disqualification periods for offenders (i.e. as a form of exposure

139 control). Other offences included speeding, unlicensed driving, using a mobile phone

140 while driving, violations of road rules and red-lighting running. In order to test for

141 statistical significant differences in these rates across the different licence/sanction

142 periods, rate ratios were calculated separately for drink-driving and general traffic

143 offence rates for:

- Licence disqualification versus pre-licence disqualification;

- Licence disqualification versus post-licence restoration; and

- Post-licence restoration versus pre-licence disqualification.

147 In order to determine the statistical significance of the rate ratios, confidence intervals

148 for all rate ratios were calculated as follows:

$$
\text { Where: } \quad S E=\sqrt{ }\left(\frac{1}{X_{1}}+\frac{1}{X_{2}}\right)
$$
period 2. 
Rate ratios were calculated and compared for each period by index offence BAC level category (Low-range - between .001 and .070; Mid-range - between .071 and .149;

157 High-range - .150 and above), gender, age group (16-24, 25-49, 50+), repeat offender 158 status (at index) and involvement in a crash at index offence.

159 The weighted mean of the rate ratios across the strata (e.g., male versus female) was 160 calculated using the Cochran-Mantel-Haenzel for incidence rates. The rate ratios for each variable stratum were then compared to the Cochran-Mantel-Haenzel rate ratio using a Chi-square test for homogeneity. The formula is as follows:

\section{Results}


175 The characteristics of the drink-driving offenders in the licence period are outlined in

176 Table 1 . The majority of offenders were male. There was a greater prevalence of first

177 time than repeat offenders. Approximately, 5\% of offenders were involved in a crash at

178 the time of their index offence.

179 Table 1: Characteristics of the drink-driving offender sample

\begin{tabular}{lcc}
\hline Characteristic & $\mathbf{N}$ & $\mathbf{\%}$ \\
\hline Gender & & \\
Male & 25,391 & 86.9 \\
Female & 3,813 & 13.1 \\
Age group & & \\
16-24 & 11,474 & 39.3 \\
$25-49$ & 15,687 & 53.7 \\
$50+$ & 2,043 & 7.0 \\
BAC level (index offence) & & \\
Low-range (between .001 and .070) & 3,269 & 11.2 \\
Mid-range (between .071 and .149) & 15,705 & 53.8 \\
High-range (.150 and above) & 4,155 & 14.2 \\
Licence type & & \\
Learner & 813 & 2.8 \\
Probationary & 8,138 & 27.9 \\
Open & 20,253 & 69.4 \\
Offender status at index & & \\
First time offender & 24,641 & 84.4 \\
Repeat offender & 4,563 & 15.6 \\
Crash at index offence & & \\
Yes & 1,540 & \\
No & 27,664 & \\
\hline
\end{tabular}

181 Table 2 shows the re-offence and crash rates (drink-driving and other) for all drinkdriving offenders. The highest rates of re-offending were in the licence period between 

restoration.

Table 2: Offence rates (per 1,000 person years) for all offenders for each licence period

\begin{tabular}{lcc}
\hline Time period & $\begin{array}{c}\text { Drink-driving } \\
\text { offences }\end{array}$ & $\begin{array}{c}\text { General traffic } \\
\text { offences }^{\mathbf{1}}\end{array}$ \\
\hline $\begin{array}{l}\text { Period between index offence and licence } \\
\text { disqualification (pre-licence disqualification) }\end{array}$ & 93.7 & 914.4 \\
Period during licence disqualification & 28.3 & 307.5 \\
Period post-licence restoration & 53.7 & 664.0 \\
\hline
\end{tabular}

${ }^{1}$ Excluding drink-driving offences

As shown in Table 3 below, all drink-driving offenders had a statistically significantly lower rate of offending (both drink-driving and other traffic offences) during licence disqualifications compared to the pre-licence disqualification and post-licence restoration periods. Also, the post-disqualification licensed period had a statistically significantly lower rate of offending compared to the pre-licence disqualification period.

Table 3: Offence rate ratios all drink-drivers

\begin{tabular}{lcc}
\hline Comparison & \multicolumn{2}{c}{ Rate ratio (95\% CI) } \\
\cline { 2 - 3 } & Drink-driving offences & Other traffic offences \\
\hline $\begin{array}{l}\text { Licence disqualification vs. Pre-licence } \\
\text { disqualification }\end{array}$ & $0.30 *(0.27-0.33)$ & $0.34^{*}(0.33-0.35)$ \\
$\begin{array}{l}\text { Licence disqualification vs. Post-licence } \\
\text { restoration }\end{array}$ & $0.53^{*}(0.49-0.57)$ & $0.46^{*}(0.45-0.48)$ \\
$\begin{array}{l}\text { Post-licence restoration vs. Pre-licence } \\
\text { disqualification }\end{array}$ & $0.57^{*}(0.53-0.62)$ & $0.73^{*}(0.71-0.74)$ \\
\hline
\end{tabular}

*Statistically significant rate ratios $(\mathrm{p}<.05)$

\section{Gender}

As shown in Table 4, males had higher rates of offending for all licence periods. The pattern of offending across periods was similar however, with both males and females having the highest rate of offending (both drink-driving and other traffic offences) in the 
pre-licence disqualification period, followed by the post-licence restoration period and

199 then the licence disqualification period.

200 Table 4: Offence rates (per 1,000 person years) by gender for each licence period

\begin{tabular}{|c|c|c|c|c|}
\hline \multirow[b]{2}{*}{ Period } & \multicolumn{2}{|c|}{ Male } & \multicolumn{2}{|c|}{ Female } \\
\hline & Drink-driving & $\begin{array}{l}\text { General } \\
\text { traffic }^{1}\end{array}$ & $\begin{array}{l}\text { Drink- } \\
\text { driving }\end{array}$ & $\begin{array}{l}\text { General } \\
\text { traffic }^{1}\end{array}$ \\
\hline $\begin{array}{l}\text { Between index offence } \\
\text { and licence } \\
\text { disqualification (pre- } \\
\text { licence disqualification) }\end{array}$ & 96.3 & 952.7 & 75.3 & 643.0 \\
\hline $\begin{array}{l}\text { During licence } \\
\text { disqualification }\end{array}$ & 29.0 & 314.2 & 23.0 & 259.4 \\
\hline Post-licence restoration & 56.2 & 690.3 & 37.3 & 493.9 \\
\hline
\end{tabular}

$201 \quad{ }^{1}$ Excluding drink-driving offences

202 Table 5 shows the rate ratios for drink-driving and other offences for each licence

203 period comparison stratified by gender. Chi-square tests for the homogeneity showed no

204 statistically significant differential effects of gender for any licence period comparisons.

205 Table 5: Offence rate ratios by gender

\section{Rate ratios $(95 \% \mathrm{CI})$}

\begin{tabular}{lcccc}
\cline { 2 - 5 } Comparison & \multicolumn{2}{c}{ Male } & \multicolumn{2}{c}{ Female } \\
\cline { 2 - 5 } & $\begin{array}{c}\text { Drink-driving } \\
\text { offences }\end{array}$ & $\begin{array}{c}\text { Other traffic } \\
\text { offences }\end{array}$ & $\begin{array}{c}\text { Drink-driving } \\
\text { offences }\end{array}$ & $\begin{array}{c}\text { Other traffic } \\
\text { offences }\end{array}$ \\
\hline $\begin{array}{l}\text { Licence } \\
\text { disqualification vs. }\end{array}$ & $0.30^{*}$ & $0.33^{*}$ & $0.31^{*}$ & $0.40^{*}$ \\
$\begin{array}{l}\text { pre-licence } \\
\text { disqualification }\end{array}$ & $(0.27-0.33)$ & $(0.32-0.34)$ & $(0.22-0.42)$ & $(0.34-0.45)$ \\
$\begin{array}{l}\text { Licence } \\
\text { disqualification vs. }\end{array}$ & $0.52^{*}$ & $0.46^{*}$ & $0.62^{*}$ & $0.53^{*}$ \\
$\begin{array}{l}\text { Post-licence } \\
\text { restoration }\end{array}$ & $(0.48-0.55)$ & $(0.45-0.47)$ & $(0.49-0.77)$ & $(0.47-0.56)$ \\
$\begin{array}{l}\text { Post-licence } \\
\text { restoration vs. Pre- }\end{array}$ & $0.58^{*}$ & $0.72^{*}$ & $0.50^{*}$ & $0.77^{*}$ \\
$\begin{array}{l}\text { licence } \\
\text { disqualification }\end{array}$ & $(0.54-0.64)$ & $(0.71-0.74)$ & $(0.38-0.64)$ & $(0.71-0.84)$ \\
\hline
\end{tabular}


208 Age

209 As shown in Table 6, those offenders aged 16-24 years had the highest rate of offending 210 in all licence periods, followed by those aged 25-49 years. The pattern of offending 211 across periods was similar however, with all age groups having the highest rate of

212 offending (both drink-driving and other traffic offences) in the period between index 213 offence and the licence disqualification, followed by the post-licence restoration period, 214 and then the licence disqualification period. 


\begin{tabular}{|c|c|c|c|c|c|c|}
\hline & \multicolumn{2}{|c|}{ 16-24 } & \multicolumn{2}{|c|}{$25-49$} & \multicolumn{2}{|c|}{$50+$} \\
\hline & $\begin{array}{c}\text { Drink-driving } \\
\text { offences }\end{array}$ & $\begin{array}{c}\text { General traffic } \\
\text { offences }^{1}\end{array}$ & $\begin{array}{c}\text { Drink-driving } \\
\text { offences }\end{array}$ & $\begin{array}{c}\text { General traffic } \\
\text { offences }^{1}\end{array}$ & $\begin{array}{c}\text { Drink-driving } \\
\text { offences }\end{array}$ & $\begin{array}{c}\text { General traffic } \\
\text { offences }^{1}\end{array}$ \\
\hline $\begin{array}{l}\text { Period between index offence } \\
\text { and licence disqualification }\end{array}$ & 97.2 & 1187.8 & 92.0 & 773.8 & 88.7 & 579.5 \\
\hline $\begin{array}{l}\text { Period during licence } \\
\text { disqualification }\end{array}$ & 35.9 & 431.6 & 24.6 & 254.6 & 20.8 & 131.9 \\
\hline Period post-licence restoration & 57.8 & 839.8 & 52.1 & 568.7 & 42.6 & 380.9 \\
\hline
\end{tabular}




\section{Rate ratios $(95 \% \mathrm{CI})$}

\begin{tabular}{|c|c|c|c|c|c|}
\hline \multicolumn{2}{|c|}{$16-24$ years } & \multicolumn{2}{|c|}{ 25-49 years } & \multicolumn{2}{|c|}{50 years +} \\
\hline $\begin{array}{c}\text { Drink-driving } \\
\text { offences }\end{array}$ & $\begin{array}{c}\text { Other traffic } \\
\text { offences }^{1}\end{array}$ & $\begin{array}{c}\text { Drink-driving } \\
\text { offences }\end{array}$ & $\begin{array}{c}\text { Other traffic } \\
\text { offences }^{1}\end{array}$ & $\begin{array}{c}\text { Drink-driving } \\
\text { offences }\end{array}$ & $\begin{array}{c}\text { Other traffic } \\
\text { offences }^{1}\end{array}$ \\
\hline $0.37^{*}$ & $0.36^{*}$ & $0.27^{*}$ & $0.33^{*}$ & $0.23 *$ & $0.23^{*}$ \\
\hline$(0.32-0.43)$ & $(0.35-0.38)$ & $(0.23-0.31)$ & $(0.31-0.34)$ & $(0.16-0.35)$ & $(0.19-0.27)$ \\
\hline $0.62 *$ & $0.51 *$ & $0.47^{*}$ & $0.45 *$ & $0.49 *$ & $0.35 *$ \\
\hline$(0.56-0.69)$ & $(0.50-0.53)$ & $(0.43-0.52)$ & $(0.43-0.46)$ & $(0.36-0.66)$ & $(0.31-0.39)$ \\
\hline $0.59 *$ & $0.71^{*}$ & $0.57 *$ & $0.73 *$ & $0.48^{*}$ & $0.66^{*}$ \\
\hline$(0.52-0.68)$ & $(0.68-0.73)$ & $(0.51-0.63)$ & $(0.71-0.76)$ & $(0.35-0.66)$ & $(0.58-0.74)$ \\
\hline
\end{tabular}

*Statistically significant rate ratios for licence periods $(\mathrm{p}<.05)$

${ }^{1}$ Excluding drink-driving offences 
227 As shown in Table 8, those offenders with a low-range index BAC had higher rates of offending across all licence periods, followed by those with a mid-range index BAC, with the lowest rates of offending for offenders with a high BAC index offence. Again, the pattern of offending was similar across licence periods with all BAC levels having the highest rate of offending (both drink-driving and other traffic offences) in the period between index offence and the licence disqualification, followed by the post-licence restoration period, and then the

231 licence disqualification period.

Table 8: Offence rates (per 1,000 person years) by BAC level for each licence period

\begin{tabular}{|c|c|c|c|c|c|c|}
\hline & \multicolumn{2}{|c|}{ Low-range } & \multicolumn{2}{|c|}{ Mid-range } & \multicolumn{2}{|c|}{ High-range } \\
\hline & $\begin{array}{c}\text { Drink-driving } \\
\text { offences }\end{array}$ & $\begin{array}{c}\text { General traffic } \\
\text { offences }^{1}\end{array}$ & $\begin{array}{c}\text { Drink-driving } \\
\text { offences }\end{array}$ & $\begin{array}{c}\text { General traffic } \\
\text { offences }^{1}\end{array}$ & $\begin{array}{c}\text { Drink-driving } \\
\text { offences }\end{array}$ & $\begin{array}{c}\text { General traffic } \\
\text { offences }^{1}\end{array}$ \\
\hline $\begin{array}{l}\text { Period between index offence } \\
\text { and licence disqualification }\end{array}$ & 90.4 & 1284.3 & 84.3 & 705.6 & 56.5 & 337.5 \\
\hline $\begin{array}{l}\text { Period during licence } \\
\text { disqualification }\end{array}$ & 49.2 & 644.1 & 21.8 & 199.8 & 15.1 & 183.3 \\
\hline Period post-licence restoration & 62.7 & 805.9 & 39.6 & 460.3 & 38.3 & 334.0 \\
\hline
\end{tabular}

${ }^{1}$ Excluding drink-driving offences 
234 Table 9 shows the rate ratios for drink-driving and other offences for each

235 comparison period stratified by BAC level at index offence. For other traffic

236 offences, Chi-square tests for the homogeneity showed some statistically significant

237 differential effects of BAC level. Specifically, low-range and high-range BAC

238 offenders had higher rate ratios for other traffic offending for the licence

239 disqualification period versus the pre-licence disqualification period $\left[\chi^{2}(2)=\right.$

$24014.18, p<.001]$. Further, for other traffic offences, high-range BAC offenders had

241 no statistically significant effect for post-licence restoration period versus the pre-

242 licence disqualification period, while low- and mid-range offenders had lower other

243 traffic offence rates during post-licence restoration period compared to the pre-

244 licence disqualification period $\left[\chi^{2}(2)=10.65, p<.001\right]$. For drink-driving

245 offences, there was a differential effect for the licence disqualification period versus

246 the post-licence restoration period $\left[\chi^{2}(2)=9.78, p=.008\right]$ with low-range BAC

247 offenders having a higher rate ratio of offending compared with mid- and high-

248 range offenders. 
Rate ratios $(95 \% \mathrm{CI})$

\begin{tabular}{|c|c|c|c|c|c|}
\hline \multicolumn{2}{|c|}{ Low-range BAC } & \multicolumn{2}{|c|}{ Mid-range BAC } & \multicolumn{2}{|c|}{ High-range BAC } \\
\hline $\begin{array}{c}\text { Drink-driving } \\
\text { offences }\end{array}$ & $\begin{array}{c}\text { Other traffic } \\
\text { offences }^{1}\end{array}$ & $\begin{array}{c}\text { Drink-driving } \\
\text { offences }\end{array}$ & $\begin{array}{c}\text { Other traffic } \\
\text { offences }^{1}\end{array}$ & $\begin{array}{c}\text { Drink-driving } \\
\text { offences }\end{array}$ & $\begin{array}{c}\text { Other traffic } \\
\text { offences }^{1}\end{array}$ \\
\hline $0.54^{*}$ & $0.50^{*}$ & $0.26^{*}$ & $0.28 *$ & $0.27^{*}$ & $0.54^{*}$ \\
\hline$(0.42-0.71)$ & $(0.47-0.54)$ & $(0.23-0.29)$ & $(0.27-0.30)$ & $(0.20-0.36)$ & $(0.48-0.61)$ \\
\hline $0.80^{*}$ & $0.78^{*}$ & $0.55^{*}$ & $0.43 *$ & $0.39 *$ & $0.53^{*}$ \\
\hline$(0.65-0.95)$ & $(0.76-0.84)$ & $(0.50-0.61)$ & $(0.42-0.45)$ & $(0.33-0.48)$ & $(0.50-0.57)$ \\
\hline $0.69 *$ & $0.63^{*}$ & $0.47 *$ & $0.65 *$ & $0.68 *$ & 1.02 \\
\hline$(0.56-0.87)$ & $(0.59-0.67)$ & $(0.42-0.52)$ & $(0.63-0.68)$ & $(0.53-0.87)$ & $(0.92-1.13)$ \\
\hline
\end{tabular}

*Statistically significant rate ratios for licence periods $(\mathrm{p}<.05)$

$251{ }^{1}$ Excluding drink-driving offences 
253 Repeat offenders had lower offence rates compared to first offenders for all periods except

254 the post-licence restoration period (Table 10). The pattern of offending across licence

255 periods, however, was consistent as per all drink-drivers and the previous comparison groups.

256 Table 10: Offence rates (per 1,000 person years) by repeat offender status at index for each 257 licence period

\begin{tabular}{lcccc}
\hline & \multicolumn{2}{c}{ First offenders } & \multicolumn{2}{c}{ Repeat offenders } \\
\cline { 2 - 5 } & $\begin{array}{c}\text { Drink-driving } \\
\text { offences }\end{array}$ & $\begin{array}{c}\text { General traffic } \\
\text { offences }^{\mathbf{1}}\end{array}$ & $\begin{array}{c}\text { Drink-driving } \\
\text { offences }\end{array}$ & $\begin{array}{c}\text { General traffic } \\
\text { offences }^{\mathbf{1}}\end{array}$ \\
\hline $\begin{array}{l}\text { Period between } \\
\text { index offence and }\end{array}$ & 97.1 & 938.7 & 78.6 & 807.8 \\
$\begin{array}{l}\text { licence } \\
\text { disqualification }\end{array}$ & 30.2 & 332.8 & & \\
$\begin{array}{l}\text { Period during } \\
\text { licence } \\
\text { disqualification }\end{array}$ & & & 22.7 & 232.2 \\
$\begin{array}{l}\text { Period post- } \\
\text { licence restoration }\end{array}$ & 53.2 & 663.3 & & \\
\hline
\end{tabular}

${ }^{1}$ Excluding drink-driving offences

Table 11 shows the rate ratios for drink-driving and other offences for each licence period comparison stratified by whether the offender was a repeat or first offender at index offence.

261 For drink-driving offences, Chi-square tests for the homogeneity showed a statistically significant effect for the licence disqualification versus the post-licence restoration period $\left[\chi^{2}\right.$ $(1)=4.50, p<.001]$. Specifically, while both groups had lower drink-driving offence rates during a disqualification in comparison with post-licence restoration, the rate ratio was lower for repeat drink-driving offenders at index offence. For other traffic offences, Chi-square tests for the homogeneity showed no statistically significant effect. 


\begin{tabular}{|c|c|c|c|c|}
\hline \multirow[b]{3}{*}{ Comparison } & \multicolumn{4}{|c|}{ Rate ratios $(95 \% \mathrm{CI})$} \\
\hline & \multicolumn{2}{|c|}{ First offenders at index } & \multicolumn{2}{|c|}{ Repeat offender at index } \\
\hline & $\begin{array}{c}\text { Drink-driving } \\
\text { offences }\end{array}$ & $\begin{array}{c}\text { Other traffic } \\
\text { offences }^{1}\end{array}$ & $\begin{array}{c}\text { Drink-driving } \\
\text { offences }\end{array}$ & $\begin{array}{c}\text { Other traffic } \\
\text { offences }^{1}\end{array}$ \\
\hline $\begin{array}{l}\text { Licence } \\
\text { disqualification vs. } \\
\text { Pre-licence } \\
\text { disqualification }\end{array}$ & $\begin{array}{c}0.31^{*} \\
(0.28-0.35)\end{array}$ & $\begin{array}{c}0.35^{*} \\
(0.34-0.37)\end{array}$ & $\begin{array}{c}0.29 * \\
(0.23-0.36)\end{array}$ & $\begin{array}{c}0.29 * \\
(0.27-0.31)\end{array}$ \\
\hline $\begin{array}{l}\text { Licence } \\
\text { disqualification vs. } \\
\text { Post-licence } \\
\text { restoration }\end{array}$ & $\begin{array}{c}0.57 * \\
(0.52-0.61)\end{array}$ & $\begin{array}{c}0.50 * \\
(0.49-0.51)\end{array}$ & $\begin{array}{c}0.40 * \\
(0.34-0.47)\end{array}$ & $\begin{array}{c}0.35^{*} \\
(0.33-0.37)\end{array}$ \\
\hline $\begin{array}{l}\text { Post-licence } \\
\text { restoration vs. Pre- } \\
\text { licence disqualification }\end{array}$ & $\begin{array}{c}0.55^{*} \\
(0.50-0.60)\end{array}$ & $\begin{array}{c}0.71^{*} \\
(0.69-0.73)\end{array}$ & $\begin{array}{c}0.83^{*} \\
(0.59-0.88)\end{array}$ & $\begin{array}{c}0.72 * \\
(0.78-0.88)\end{array}$ \\
\hline
\end{tabular}

271 *Statistically significant rate ratios for licence periods $(\mathrm{p}<.05)$

$272 \quad{ }^{1}$ Excluding drink-driving offences

273 Crash at index offence

274 Those who were involved in a crash at the same time as their index offence had lower offence

275 rates for all licence periods compared to those that did not have a crash at index offence

276 (Table 12). The pattern of results across licence periods was somewhat consistent. However,

277 for general traffic offences, the offence rate was highest in the post-licence restoration period

278 for those who had a crash at index offence (although still a lower rate than those who were

279 not involved in a crash at index). 
Table 12: Offence rates (per 1,000 person years) by crash at index offence status for each licence period

\begin{tabular}{lcccc}
\hline & \multicolumn{2}{c}{ Crash involved } & \multicolumn{2}{c}{ Non-crash involved } \\
\cline { 2 - 5 } & $\begin{array}{c}\text { Drink-driving } \\
\text { offences }\end{array}$ & $\begin{array}{c}\text { General traffic } \\
\text { offences }^{\mathbf{1}}\end{array}$ & $\begin{array}{c}\text { Drink-driving } \\
\text { offences }\end{array}$ & $\begin{array}{c}\text { General traffic } \\
\text { offences }^{\mathbf{1}}\end{array}$ \\
\hline $\begin{array}{l}\text { Period between } \\
\text { index offence and }\end{array}$ & 45.0 & 460.5 & 99.0 & 963.5 \\
$\begin{array}{l}\text { licence } \\
\text { disqualification }\end{array}$ & 14.4 & 179.6 & 29.2 & \\
$\begin{array}{l}\text { Period during } \\
\text { licence } \\
\text { disqualification }\end{array}$ & & & & 315.6 \\
$\begin{array}{l}\text { Period post- } \\
\text { licence restoration }\end{array}$ & 41.7 & 542.5 & 54.3 & \\
\hline
\end{tabular}

${ }^{1}$ Excluding drink-driving offences

284 Table 13 shows the rate ratios for drink-driving and other offences for each licence period comparison stratified by whether the offender was involved in a crash at the index offence or not. For other traffic offences, Chi-square tests for the homogeneity showed a statistically significant effect for the post-licence restoration period versus the pre-licence disqualification period $\left[\chi^{2}(1)=14.51, p<.001\right]$. Specifically, those offenders who were involved in a crash at the time of their index offence had a higher other offence rate during the post-licence restoration period compared to the pre-licence disqualification period, while those who were not involved in a crash had lower other offence rates during the post-licence restoration period. There were no other differential effects based on crash involvement at index offence. 


\begin{tabular}{|c|c|c|c|c|}
\hline \multirow[b]{3}{*}{ Comparison } & \multicolumn{4}{|c|}{ Rate ratios $(95 \% \mathrm{CI})$} \\
\hline & \multicolumn{2}{|c|}{ Crash involved at index } & \multicolumn{2}{|c|}{ Not crash involved at index } \\
\hline & $\begin{array}{c}\text { Drink-driving } \\
\text { offences }\end{array}$ & $\begin{array}{c}\text { Other traffic } \\
\text { offences }^{1}\end{array}$ & $\begin{array}{c}\text { Drink-driving } \\
\text { offences }\end{array}$ & $\begin{array}{c}\text { Other traffic } \\
\text { offences }^{1}\end{array}$ \\
\hline $\begin{array}{l}\text { Licence } \\
\text { disqualification vs. } \\
\text { Pre-licence } \\
\text { disqualification }\end{array}$ & $\begin{array}{c}0.32 * \\
(0.20-0.53)\end{array}$ & $\begin{array}{c}0.39 * \\
(0.34-0.45)\end{array}$ & $\begin{array}{c}0.29 * \\
(0.27-0.33)\end{array}$ & $\begin{array}{c}0.33^{*} \\
(0.32-0.34)\end{array}$ \\
\hline $\begin{array}{l}\text { Licence } \\
\text { disqualification vs. } \\
\text { Post-licence } \\
\text { restoration }\end{array}$ & $\begin{array}{c}0.35^{*} \\
(0.23-0.51)\end{array}$ & $\begin{array}{c}0.33^{*} \\
(0.30-0.37)\end{array}$ & $\begin{array}{c}0.54 * \\
(0.50-0.58)\end{array}$ & $\begin{array}{c}0.47^{*} \\
(0.46-0.48)\end{array}$ \\
\hline $\begin{array}{l}\text { Post-licence } \\
\text { restoration vs. } \\
\text { Pre-licence } \\
\text { disqualification }\end{array}$ & $\begin{array}{c}0.93 \\
(0.63-1.36)\end{array}$ & $\begin{array}{c}1.18^{*} \\
(1.05-1.32)\end{array}$ & $\begin{array}{c}0.55^{*} \\
(0.51-0.60)\end{array}$ & $\begin{array}{c}0.70 * \\
(0.68-0.71)\end{array}$ \\
\hline
\end{tabular}

*Statistically significant rate ratios for licence periods $(\mathrm{p}<.05)$

${ }^{1}$ Excluding drink-driving offences

\section{Discussion}

The primary aims of this project were to determine: (a) whether drink-drivers’ re-offence rates differed during the period between offence incidence and licence disqualification (prelicence disqualification), the period of licence disqualification and the post-licence restoration period; and (b) identify if there are any differential effects of licence disqualification on reoffence rates based on BAC, gender, age, repeat offender status and crash involvement at the time of offence. The offences analysed were drink-driving offences and other traffic offences. Key findings that emerged will be sequentially discussed below.

In regards to the rate of offending, the highest rate of offending (both drink-driving and other traffic offences) was during the period between the index offence and the commencement of the licence disqualification (pre-licence disqualification). This is a key finding to emerge from the study that needs to be re-examined with other traffic offence data in other jurisdictions in the future. In Victoria, we found that offenders are at the highest risk of 
drink-driving (or committing other traffic offences) after they have been apprehended, but before they receive the corresponding sanctions. This finding supports the assertion that the celerity of punishment (in regards to deterrence models) is an important factor in maximising a deterrent effect. However, the celerity of sanction application is commonly overlooked with deterrence-based research (Freeman, 2004), except for preliminary research that has focused on the positive impact of changes to administrative suspension laws (McArthur, \& Kraus, 1999; Voas, Tippets \& Fell, 2000; Zador et al., 1988). For example, Wagenaar \& Maldonado-Molina (2007) reviewed the impact of mandatory preconviction licence suspension laws in 46 American states and reported the policy had a statistically significant reduction in alcohol-related crash involvement. This is despite models of learning and experimental psychology reinforcing that the time between stimulus and response is vital for learning new behaviours (Nagin \& Pogarsky, 2001).

More encouragingly, drink-driving offenders had statistically significantly lower rates of offending (both drink-driving and other traffic offences) during disqualification periods compared to the pre-licence disqualification and post-licence restoration periods. In regards to first time versus recidivist offenders, both groups had lower drink-driving offence rates during disqualification in comparison with post-licence restoration. High BAC offenders also had low rates of re-offending during disqualification relative to other BAC offender groups contrary to perceptions that they are less responsive to countermeasures. This finding is consistent with a large body of research that has generally demonstrated licence disqualification periods to be one of the most effective methods for reducing further drinkdriving offences (Jones \& Lacey, 1991; McArthur \& Kraus, 1999; Nichais \& Ross, 1991; Sadler \& Perrine, 1984; Wagenaar, Zoeck, Williams \& Hingson, 1995). In fact, compared to other sanctions, disqualification periods have proven to be the most effective short-term 
countermeasure that can be applied to drink-drivers (Nichais \& Ross, 1991; Sadler \& Perrine, 1984; Siskind, 1996; Watson, 1998). The current finding is particularly encouraging in relation to high BAC and recidivist offenders, as ongoing questions have remained regarding the efficacy of applying sanctions to persistent offenders (Freeman, Liossis, \& David, 2006) and high BAC offenders who are perceived as difficult to influence - in contrast this study clearly shows an impact of licence sanction on drink driving offenders during and following licence disqualification for most detected offenders. However, there still was evidence that a small minority of individuals were detected again for drink-driving even while disqualified from driving as found for $4 \%$ of the sample. That is, they combined drink-driving with unlicensed driving, demonstrating that licence disqualification does not have a positive impact on all individuals. This is again consistent with research that has reported unlicensed driving is often combined with other illegal behaviours such as drink-driving (Griffin \& DeLaZerda, 2000; Watson, 2004).

Further analyses revealed that there was in fact a greater effect of the disqualification on repeat drink-driving offenders compared to first time offenders, as well as for high BAC offenders. This is contrary to previous research that has demonstrated that licence sanctions are least effective for repeat offenders (Beirness et al., 1997; Coben \& Larkin, 1999). Different theories can account for this finding. Firstly, it is possible that (for the current sample) repeat offenders reduced their frequency of driving to a greater extent than first time objective certainty. Another hypothesis proposed by Pogarsky and Piquero (2003), that needs to be further investigated, is whether first time offenders experience a "resetting effect" after apprehension, whereby offenders believe they are less likely to be apprehended again soon after coming in contact with the police. However, the above results should be interpreted with 
caution as there is no comparison group; so, for example, it is not possible to tell if a disqualification period is the most effective sanction compared to any other sanction (e.g., immediate interlock condition).

Post Licence periods

Another key finding was that there was also a statistically significantly lower rate of offending (both drink-driving and other traffic) in the post-licence restoration period compared to the pre-licence disqualification period (40\% lower), both for first time and repeat drink-drivers. In regards to first time offenders, this is consistent with previous research that has demonstrated licence disqualifications have a specific deterrent effect post licence restoration. (Homel, 1988; Siskind, 1996). That is, convicted offenders are less likely to re-offend due to experience with the consequences of penalties. The results were also positive for repeat offenders. While there has generally been consensus in the literature that the application of legal sanctions alone does not produce long-term behaviour change for this group (Ahlin, Rauch, Zador, Baum, \& Duncan, 2002; Beirness et al., 1997; Brewer et al., 1994; Frank, Raub, Lucke, \& Wark, 2002; Homel, 1988; Marques, Voas, \& Hodgins, 1998; Yu, 2000), the current study has provided evidence that disqualifications can have a corrective effect on tendencies to drink and drive among recidivist offenders. However, it should be noted, that as there was no control group for this analysis (i.e., offenders who did not have licence sanctions applied) the changes in offending rates may have been due in part to other influences (e.g., enforcement practices). Furthermore, while the offending rate decreased post-sanction, it is not possible to tell if this is a genuine positive effect of experiencing the sanction or if, for example, offenders improved their ability to avoid detection once they have experienced this sanction. In regards to the latter, previous research has found that offenders, particularly repeat offenders, can drink and drive on numerous occasions whilst avoiding detection (Wiliszowski, Murphy, Jones, \& Lacey, 1996). For 
example, Smith (2003) interviewed a small sample of repeat offenders who reported regularly drink-driving whilst avoiding apprehension (e.g., ratios up to 100:1) as well as actively attempting to evade police enforcement (e.g., Random Breath Testing).

In regards to the differential offence rate effects, the second aim of the study, males had higher rates of drink-driving offending for all licence periods, which is consistent with previous research that has demonstrated that males are disproportionately represented in drink-driving statistics (Beirness et al., 1997; Stewart et al., 2004; Voas \& Tippetts, 2002). However, while absolute rates of offending differed by gender and between age groups, the pattern of rates of offending across the three study periods for each of these subgroups was similar. No subgroups were more likely to drink and drive (or commit other traffic offences) in the pre-licence disqualification period, the post-licence restoration period or the licence disqualification period. The current findings indicate that disqualifications can have a positive effect on both genders as well as motorists of all ages. This is one of the first studies to specifically examine offence rates with respect to age and gender, and therefore further research is required to confirm this finding.

There were however, some statistically significant differential effects of BAC level and of repeat offender status. While all BAC groups demonstrated a reduction in drink-driving offence rates during the licence disqualification period compared to the post-licence restoration period, low-range BAC offenders had a higher offence rate ratio compared with mid- and high-range offenders. Therefore, the disqualification period appeared to have a 407 lesser impact on the low-range BAC group for drink-driving and other traffic offences. This could be considered an unexpected finding, as a higher range BAC could be considered evidence of an alcohol problem, which has been demonstrated to be a significant predictor of recidivism (Freeman et al., 2006). While it remains unclear why this was found, two possible 
explanations can be proposed. Firstly, it may be because the disqualification period this group received was not as severe (compared to high BAC range offenders) and thus, less of a specific deterrent effect was experienced for the less severe sanction. Secondly, this group may have experienced less of an overall experiential effect (e.g., number of times exposed to punishment), and thus, have yet to be sufficiently deterred from drink-driving. Further research is required to determine the significance of this finding, as it has historically been hypothesised that low-range BAC groups predominantly involve social drinkers who may make a judgement error in their decision to drive after drinking (Ferguson et al., 1999; Howard \& McCaughrin, 1996). As a result, these drivers are usually deterred from committing further offences by their experience of both formal and informal sanctions such as fines and licence loss, as well as peer disapproval from friends and family (Ferguson et al., 1999). However, some low-range BAC offenders (apprehended in the morning) may have consumed large quantities of alcohol the night before, and this phenomenon also deserves further exploration.

There were also some differential effects for other traffic offending. High-range BAC offenders had no statistically significant effect for post-licence restoration period versus the pre-licence disqualification period, while low- and mid-range offenders had lower offence rates during the post-licence restoration period compared to the pre-licence disqualification period.

Importantly, offenders who were involved in a crash at the time of their index offence had a higher general traffic offending rate during the post-licence restoration period compared to the pre-licence disqualification period, while those who were not involved in a crash had lower offence rates during the post-licence restoration period compared to the pre-licence disqualification period. It is not clear whether this is a direct result of the disqualification or a bias of having experienced a crash. For example, crash involved offenders may be injured 
and unable to drive, or without a vehicle in the immediate period following the crash. These factors may have a greater impact on influencing driving behaviour than the actual sanction.

There were a number of limitations associated with this study that need to be considered.

Firstly, as with any study of this nature, the sample only includes those who are caught for an offence. It is possible that some offenders are not captured as they are able to avoid detection. For example, an earlier study by Voas (1982) reported that the drinking driver is arrested once out of every 5000 miles (approximately 8,000 kilometres) driven under the influence of alcohol. A similar estimation in the Australian context offered by Homel, Carseldine, and Kearns (1988) suggested that only $0.5 \%$ to $1.5 \%$ of intoxicated drivers are detected by the police at any one time. While more recent calculations are not available, the deleterious impact of 'punishment avoidance' on intentions to re-offend is well documented (Freeman \& Watson, 2006; Watling, Freeman, Palk, \& Davey, 2011). In the current context, this would result in an under-estimate of the drink-driving problem. It is also possible that particular types of offenders are better at avoiding detection and thus the study may not capture all types of drink-driving offenders. Some offenders within the study sample may also avoid detection some of the time or even improve their avoidance over time. This may impact on the re-offence rates for some of these offenders and bias the results to some extent if particular types of offenders (e.g., repeat offenders) become better at detection avoidance than others.

It should be noted that the BAC level for classification may lack some sensitivity to offender differences within BAC groups. While BAC groupings in this study were consistent with the legislative levels relating to sanctions as well as reflecting escalating trauma risk with higher BAC levels, it could be argued that there may be some distinct differences within these level classifications that were not able to be explored. For example, there may be little difference between an offender with a BAC of .14 and one with a BAC of .15 (in different categories) 
and a large difference between a person with a BAC of .08 and one with a BAC of .12 (in the same category for some analyses). Research suggests that drink-driving offenders are not a homogenous group even within these categorisations of low-, mid- and high-range BACs (Fetherston, Lenton, \& Cercarelli, 2002; Nadeau, 2002; Nochajski \& Wieczorek, 2000). Thus, differences explored between these groups may lack sensitivity. Additionally, some of the study's findings may be unique to the data set (and time period), and thus, the study methodology needs to be implemented with different datasets.

The present study has provided further confirmatory evidence that licence disqualification periods are effective at reducing drink-driving offending, both while drivers are disqualified as well as post relicensing. Encouragingly, the application of the sanction also had a positive effect on general traffic offending, recidivist drink-drivers and the effectiveness of the approach was not diluted by gender or age group. High BAC offenders had lower re-offence rates than moderate BAC offenders who both had lower rates than low BAC offenders. However, the study identified a significant area of concern. Specifically, the highest rate of offending (both for drink-driving and other traffic offences) was during the pre-licence disqualification period, which suggests offenders are particularly vulnerable to drink and drive whilst waiting to be sanctioned. There is a need to develop effective methods to deal with offenders when they are first apprehended, including consideration of immediate licence disqualification which has been shown to be effective in studies where such an administrative sanction has been applied and evaluated (National Highway Traffic Safety Administration, 2014). An additional method may involve a brief behaviour change intervention program, which has previously been suggested in Shults et al (2001) review of drink driving countermeasures. The importance for early intervention is also evident in the corresponding offending histories of motorists involved in alcohol-related crashes, which may again be utilised as a screening tool for referral to additional services. 
The findings of this study show that the application of licence disqualification periods for drink-drivers of all types appears to be an effective response to improve road safety. The study was able to identify areas of opportunity where countermeasures could be applied to further improve offenders’ compliance with BAC limits, specifically the period immediately following police detection, compliance by lower BAC and first offenders, and following the licence disqualification period.

\section{Acknowledgements}

The authors would like to acknowledge that this study was part of a larger funded project by VicRoads. We would like to thank VicRoads and John Catchpole of ARRB Group Ltd for the preparation and provision of data.

\section{References}

Ahlin, E., Rauch, P., Zador, P., Baum, H., \& Duncan, D. (2002). Social bonds in an ignition interlock license restriction program in Maryland. Paper presented at the Proceedings of the 16th international conference on alcohol, drugs and traffic safety.

Beirness, D. J., Mayhew, D. R., \& Simpson, H. M. (1997). DWI repeat offenders: A review and synthesis of the literature.

Boorman, M. (2012). Victoria Police: The drink and drug driving enforcement link to public health. Paper presented at the The First International Conference on Law Enforcement and Public Health, Melbourne, Australia.

Brewer, R. D., Morris, P. D., Cole, T. B., Watkins, S., Patetta, M. J., \& Popkin, C. (1994). The risk of dying in alcohol-related automobile crashes among habitual drunk drivers. New England Journal of Medicine, 331(8), 513-517.

Coben, J. H., \& Larkin, G. L. (1999). Effectiveness of ignition interlock devices in reducing drunk driving recidivism. American Journal of Preventive Medicine, 16(1), 81-87. 
Coroners Prevention Unit. (2013). Presence of alcohol and drugs amongst deaths from onroad transport crashes in Victoria 2008 - 2011: Coroners Court of Victoria.

Ferguson, M., Sheehan, M. C., Davey, J. D., \& Watson, B. C. (1999). Drink driving rehabilitation: the present context: Australian Transport Safety Bureau.

Fetherston, J., Lenton, S., \& Cercarelli, L. R. (2002). The repeat drink drivers study: National Drug Research Institute, Curtin University of Technology.

Frank, J. F., Raub, R., Lucke, R. E., \& Wark, R. I. (2002). Illinois ignition interlock evaluation. Paper presented at the Proceedings of the 16th International Conference on Alcohol, Drugs and Traffic Safety.

Freeman, J. (2004). Influencing recidivist drink drivers' entrenched behaviours: the selfreported outcomes of three countermeasures.

Freeman, J., Armstrong, K., Truelove, V., Szogi, E. Left on the Side of the Road? (2015). A Review of Deterrence-based Theoretical Developments in Road Safety. Peer Reviewed proceedings of the Road Safety Research, Policing and Education Conference, Gold Coast.

Freeman, J., Liossis, P., \& David, N. (2006). Deterrence, defiance and deviance: An investigation into a group of recidivist drink drivers' self-reported offending behaviours. Australian \& New Zealand Journal of Criminology, 39(1), 1-19.

Freeman, J., \& Watson, B. (2006). An application of Stafford and Warr's reconceptualisation of deterrence to a group of recidivist drink drivers. Accident Analysis \& Prevention, 38(3), 462-471.

Griffin, L. I., \& DeLaZerda, S. (2000). Unlicensed to kill: AAA Foundation for Traffic Safety Washington, DC.

Homel, R. (1988). Policing and punishing the drinking driver. A study of specific and general deterrence: New York: Springer-Verlag. 
Homel, R., Carseldine, D., \& Kearns, I. (1988). Drink-driving countermeasures in Australia. Alcohol, drugs \& driving.

Howard, D. L., \& McCaughrin, W. C. (1996). The treatment effectiveness of outpatient substance misuse treatment organizations between court-mandated and voluntary clients. Substance use \& misuse, 31(7), 895-926.

Jones, R. K., \& Lacey, J. H. (1991). Review of the Literature Evaluating the Effect of Countermeasures to Reduce Alcohol Impaired Driving (1980-1989).: Individual Analyses and Assessments: National Highway Traffic Safety Administration.

Marques, P. R., Voas, R. B., \& Hodgins, D. (1998). Vehicle interlock programs: Protecting the community against the drunk driver. Journal of Prevention \& Intervention in the Community, 17(1), 31-44.

McArthur, D. L., \& Kraus, J. F. (1999). The specific deterrence of administrative per se laws in reducing drunk driving recidivism. American journal of preventive medicine, 16(1), $68-75$

Nadeau, L. (2002). Are there better ways to predict recidivism? Paper presented at the Proceedings International Council on Alcohol, Drugs and Traffic Safety Conference.

Nagin, D. S., \& Pogarsky, G. (2001). Integrating celerity, impulsivity, and extralegal sanction threats into a model of general deterrence: Theory and evidence. Criminology, 39, 865.

National Highway Traffic Safety Administration. (2014). Countermeasures that work: a highway safety countermeasure guide for state highway safety offices. Washington, DC: National Highway Traffic Safety Administration; 2016.

Nichols, J., \& Ross, H. (1991). The effectiveness of legal sanctions in dealing with drinking drivers. Journal of safety research, 22(2), 117. 
559 Nochajski, T., \& Wieczorek, W. (2000). Driver characteristics as a function of DWI history. Alcohol, drugs and traffic safety-T2000, Stockholm: Ekonomi-Print.

561

Peck, R. C. (1991). The general and specific deterrent effects of DUI sanctions: A review of California's experience. Alcohol, Drugs \& Driving.

Pogarsky, G., \& Piquero, A. R. (2003). Can punishment encourage offending? Investigating the "resetting” effect. Journal of Research in Crime and Delinquency, 40(1), 95-120.

Ross, H. (1991). License deprivation as a drunk-driver sanction. Alcohol, Drugs \& Driving.

Sadler, D., \& Perrine, M. (1984). An evaluation of the california drunk driving countermeasure system, volume 2: the long term traffic safety impact of a pilot alcohol abuse treatment as an alternative to license suspensions.

Shults, R. A., Elder, R. W., Sleet, D. A., Nichols, J. L., Alao, M. O., Carande-Kulis, V. G., Thompson, R. S. (2001). Reviews of evidence regarding interventions to reduce alcohol-impaired driving. American Journal of Preventive Medicine, 21(4), 66-88.

Siskind, V. (1996). Does license disqualification reduce reoffence rates? Accident Analysis \& Prevention, 28(4), 519-524.

Smith, K. P. (2003). A qualitative study of deterrence and deviance in a group of recidivist drink drivers: University of Canberra.

Stewart, S., Boase, P., \& Lamble, R. (2004). Criminal profiles of drinking drivers in Ontario. Paper presented at the International Conference on Traffic and Transport Psychology.

Voas, R. B. (1982). Drinking and driving: Scandinavian laws, tough penalties and United States alternatives.

Voas, R. B., \& Tippetts, A. S. (2002). BACs of US drivers in fatal crashes: have they changed in the last 20 years. Paper presented at the Proceedings of the 16th international conference on alcohol, drugs and traffic safety. 
583

584

585

586

587

588

589

590

591

592

593

594

595

596

597

598

599

600

601

602

603

604

Voas, R., Tippetts, S., \& Fell, J. (2000). The relationship of alcohol safety laws to drink drivers in fatal crashes. Accident Analysis and Prevention, 32, 483-492.

Wagenaar, A.C., \& Maldonado-Molina, M.M. (2007). Effects of drivers' license suspension policies on alcohol-related crash involvement: long-term follow-up in forty-six states. Alcoholism: Clinical and Experimental Research, 31(8), 1399-1406.

Wagenaar, A.C., Zobeck, T., Williams, G., \& HIngson, R. (1995). Methods used in studies of drink-drive control effects: a meta-analysis of the literature from 1960-1991. Accident Analysis and Prevention, 27(3), 307-316.

Watling, C. N., Freeman, J. E., Palk, G. R., \& Davey, J. D. (2011). Sex, drugs, and deterrence: applying Stafford and Warr's reconceptualization of deterrence theory to drug driving across the genders. Psychology of Punishment, 57-71.

Watson, B. (1998). The effectiveness of drink driving licence actions, remedial programs and vehicle-based sanctions.

Watson, B. (2004). The psychosocial characteristics and on-road behaviour of unlicensed drivers. Queensland University of Technology.

Wiliszowski, C., Murphy, P., Jones, R., \& Lacey, J. (1996). Determine reasons for repeat drinking and driving.

Yu, J. (2000). Punishment and alcohol problems: Recidivism among drinking-driving offenders. Journal of Criminal Justice, 28(4), 261-270.

Zador, P.K.; Lund, A.K.; Field M., et al (1988). Alcohol-Impaired Driving Laws and Fatal Crash Involvement. Washington, DC: Insurance Institute for Highway Safety. 